\title{
Bifurcations of Control Systems: A View from Control Flows
}

\author{
Fritz Colonius ${ }^{1}$ and Wolfgang Kliemann ${ }^{2}$ \\ 1 Institut für Mathematik, Universität Augsburg, 86135 Augsburg, Germany \\ colonius@math.uni-augsburg.de \\ 2 Department of Mathematics, Iowa State University, Ames IA 50011, U.S.A \\ kliemann@iastate.edu
}

\section{Introduction}

The purpose of this paper is to discuss bifurcation problems for control systems viewing them as dynamical systems, i.e., as control flows. Here open loop control systems are considered as skew product flows where the shift along the control functions is part of the dynamics.

Basic results from this point of view are contained in the monograph [8]. In the present paper we survey recent results on bifurcation problems-some new results are included and a number of open problems is indicated. Pertinent results from [8] are cited if necessary for an understanding.

We consider control systems in $\mathbb{R}^{d}$ of the form

$$
\dot{x}(t)=f(\alpha, x(t), u(t)), u \in \mathcal{U}=\left\{u: \mathbb{R} \rightarrow \mathbb{R}^{m}, u(t) \in U \text { for } t \in \mathbb{R}\right\},
$$

where the control range $U$ is a subset of $\mathbb{R}^{m}$ and $\alpha \in A \subset \mathbb{R}$ denotes a bifurcation parameter. For simplicity we assume that for every initial condition $x(0)=x_{0} \in \mathbb{R}^{d}$ and every admissible control function $u$ a unique global solution $\varphi^{\alpha}\left(t, x_{0}, u\right), t \in \mathbb{R}$, exists. If the dependence on $\alpha$ is irrelevant, we suppress $\alpha$ in the notation.

As for differential equations, it is relevant to discuss qualitative changes in the system behavior when $\alpha$ is varied. Such problems have found much interest in recent years; see e.g. the contributions in this volume or in [7]. The bifurcation theory developed here concerns open loop control system. Based on the concept of the associated control flow, changes in the controllability behavior come into focus. It turns out that the difference between controllability and chain controllability (which allows for arbitrarily small jumps) is decisive for our analysis. Since we discuss open loop systems with restricted control values, feedback transformations will not be allowed; this is in contrast to classical concepts of normal forms in control theory. In particular, this is a notable difference to the bifurcation and normal form theory developed recently by A. Krener and W. Kang; see, in particular, Kang [21]. 
The contents are as follows: In Section 2 we introduce our framework. Section 3 discusses bifurcation from a singular point, i.e., a point in the state space that remains fixed under all controls; here also an approach to normal forms is discussed. Section 4 treats bifurcations from invariant control sets.

Notation: For $A, B \subset \mathbb{R}^{d}$ the distance of $A$ to $B$ is

$$
\operatorname{dist}(A, B)=\sup _{a \in A} \inf _{b \in B}|a-b|
$$

and the Hausdorff distance is $\mathrm{d}_{H}(A, B)=\max (\operatorname{dist}(A, B), \operatorname{dist}(B, A))$. The topological closure and interior of a set $A$ are $\operatorname{denoted~by~} \operatorname{cl} A$ and $\operatorname{int} A$, respectively.

\section{Control Flows}

System (1) may be viewed as a family of ordinary differential equations indexed by $u \in \mathcal{U}$. Since they are non-autonomous, they do not define a flow or dynamical system. In the theory of non-autonomous differential equations there is the classical device to embed such an equation into a flow by considering all time shifts of the right hand side and to include the possible right hand sides into the state. In the context of uniformly continuous time dependence this goes back to Bebutov [3]; more recently, such constructions have been used extensively by R. Johnson and others in the context of non-autonomous (linear) control systems (e.g. Johnson and Nerurkar [19]); see also Grüne [16] for a different discussion emphasizing numerical aspects. Here, however, we will stick to autonomous control systems and only consider the time-dependence stemming from the control functions. Introduce the shift

$$
\left(\theta_{t} u\right)(\tau)=u(t+\tau), \tau \in \mathbb{R},
$$

on the set of control functions. One immediately sees that the map

$$
\Phi:\left(t, u, x_{0}\right) \mapsto\left(\theta_{t} u, \varphi\left(t, x_{0}, u\right)\right)
$$

defines a flow $\Phi$ on $\mathcal{U} \times \mathbb{R}^{d}$ : Abbreviating $\Phi_{t}=\Phi(t, \cdot, \cdot)$, one has

$$
\Phi_{0}=\mathrm{id} \text { and } \Phi_{t+s}=\Phi_{t} \circ \Phi_{s}
$$

Since the state space is infinite-dimensional, additional topological requirements are needed. We require that $\mathcal{U}$ is contained in $L_{\infty}\left(\mathbb{R}, \mathbb{R}^{m}\right)$. This gives a reasonable framework, if $U \subset \mathbb{R}^{m}$ is compact and convex and the system is control affine, i.e.,

$$
f(\alpha, x, u)=f_{0}(\alpha, x)+\sum_{i=1}^{m} u_{i} f_{i}(\alpha, x) .
$$


Then the flow $\Phi$ is continuous and $\mathcal{U}$ becomes a compact metric space in the weak* topology of $L_{\infty}\left(\mathbb{R}, \mathbb{R}^{m}\right)$ (cp. [8, Lemma 4.2.1 and Lemma 4.3.2]). We refer to system (1) with right hand side given by (2) for some $\alpha \in A$ as system $(2)^{\alpha}$; similarly, we denote objects corresponding to this system by a superfix $\alpha$. We assume that $0 \in \operatorname{int} U$. Throughout this paper we remain in this framework.

Remark 1. The class of systems can be extended, if-instead of the shift along control functions-the shift along the corresponding time dependent vector fields is considered; cp. [10] for a brief exposition.

A control set $D$ is a maximal controlled invariant set such that

$$
D \subset \mathrm{clO}^{+}(x) \text { for all } x \in D .
$$

Here $\mathcal{O}^{+}(x)=\{\varphi(t, x, u), u \in \mathcal{U}$ and $t \geq 0\}$ denotes the reachable set from $x$. A control set $C$ is called an invariant control set if $\mathrm{cl} C=\mathrm{clO}^{+}(x)$ for all $x \in C$. Often we will assume that local accessibility holds, i.e., that the small time reachable sets in forward and backward time $\mathcal{O}_{\leq T}^{+}(x)$ and $\mathcal{O}_{\leq T}^{-}(x)$, respectively, have nonvoid interiors for all $x$ and all $T>0$. Then $\operatorname{int} D \subset \mathcal{O}^{+}(x)$ for all $x \in D$. Local accessibility holds, if

$$
\operatorname{dim} \mathcal{L} \mathcal{A}\left\{f_{0}+\sum_{i=1}^{m} u_{i} f_{i},\left(u_{i}\right) \in U\right\}(x)=d \text { for all } x \in \mathbb{R}^{d} .
$$

We also recall that a chain control set $E$ is a maximal subset of the state space such that for all $x \in E$ there is $u \in \mathcal{U}$ with $\varphi(t, x, u) \in E$ for all $t \in \mathbb{R}$ and for every two elements $x, y \in E$ and all $\varepsilon, T>0$ there are $x_{0}=x, x_{1}, \ldots, x_{n}=y$ in $\mathbb{R}^{d}, u_{0}, \ldots, u_{n} \in \mathcal{U}$ and $T_{0}, \ldots, T_{n-1}>T$ with $\mathrm{d}\left(\varphi\left(T_{i}, x_{i}, u_{i}\right), x_{i+1}\right)<\varepsilon$. Every control set with nonvoid interior is contained in a chain control set; chain control sets are closed if they are bounded.

Compact chain control sets $E$ uniquely correspond to compact chain recurrent components of the control flow via

$$
\mathcal{E}=\left\{(u, x) \in \mathcal{U} \times \mathbb{R}^{d}, \varphi(t, x, u) \in E \text { for all } t \in \mathbb{R}\right\} .
$$

Control sets $D$ with nonvoid interior uniquely correspond to maximal topologically transitive sets (such that the projection to $\mathbb{R}^{d}$ has nonvoid interior) via

$$
\mathcal{D}=\operatorname{cl}\left\{(u, x) \in \mathcal{U} \times \mathbb{R}^{d}, \varphi(t, x, u) \in \operatorname{int} D \text { for all } t \in \mathbb{R}\right\} .
$$

It turns out that for parameter-dependent systems, control sets and chain control sets have complementary semicontinuity properties; see [9].

Theorem 1. Consider parameter-dependent system (2) and fix $\alpha_{0} \in A$.

(i) Let $D^{\alpha_{0}}$ be a control set with compact closure of (2) ${ }^{\alpha_{0}}$, and assume that system (2) ${ }^{\alpha_{0}}$ satisfies accessibility rank condition (4) on $\operatorname{cl} D^{\alpha_{0}}$. Then for $\alpha$ 
near $\alpha_{0}$ there are unique control sets $D^{\alpha}$ of (2) ${ }^{\alpha}$ such that the map $\alpha \longmapsto \operatorname{cl} D^{\alpha}$ is lower semicontinuous at $\alpha=\alpha_{0}$.

(ii) Let $K \subset \mathbb{R}^{d}$ be compact and suppose that for $\alpha$ near $\alpha_{0}$ the chain control sets $E^{\alpha}$ with $E^{\alpha} \cap K \neq \varnothing$ of (2) ${ }^{\alpha}$ are contained in $K$. Then $\alpha \mapsto E^{\alpha}$ is upper semicontinuous at $\alpha_{0}$ in the sense that

$$
\limsup _{\alpha \rightarrow \alpha_{0}} E^{\alpha}=\left\{x \in M, \begin{array}{c}
\text { there are } \alpha_{k} \rightarrow \alpha_{0} \text { and } \\
x^{\alpha_{k}} \in E^{\alpha_{k}} \text { with } x^{\alpha_{k}} \rightarrow x
\end{array}\right\} \subset \bigcup E^{\alpha_{0}},
$$

where the union is taken over the chain control sets $E^{\alpha_{0}} \subset K$ of (2) ${ }^{\alpha_{0}}$.

(iii) Let $D^{\alpha_{0}}$ be a control set of (2) ${ }^{\alpha_{0}}$ with $\alpha_{0} \in A$, and assume that system (2) ${ }^{\alpha_{0}}$ satisfies accessibility rank condition (4) on $\mathrm{cl} D^{\alpha_{0}}$. Let $E^{\alpha}$ be the chain control set containing the control set $D^{\alpha}$ (given by (i)) and assume that $\operatorname{cl} D^{\alpha_{0}}=E^{\alpha_{0}}$. Then the control sets $D^{\alpha}$ depend continuously on $\alpha$ at $\alpha=\alpha^{0}$,

$$
\lim _{\alpha \rightarrow \alpha_{0}} \operatorname{cl} D^{\alpha}=\lim _{\alpha \rightarrow \alpha_{0}} E^{\alpha}=\operatorname{cl} D^{\alpha_{0}}=E^{\alpha_{0}} .
$$

Remark 2. Gayer [14] shows that (i) in the previous theorem remains true if (instead of $\alpha$-dependence) the control range depends lower semicontinuously on a real parameter $\rho$.

Thus a chain control set which is the closure of a control set with nonvoid interior depends continuously on parameters. This equivalence of controllability and chain controllability may be interpreted as a structural stability property of control systems. Hence it is important to study when chain control sets coincide with the closures of control sets.

In order to allow for different maximal amplitudes of the inputs, we consider admissible controls in $\mathcal{U}^{\rho}=\left\{u \in L_{\infty}\left(\mathbb{R}, \mathbb{R}^{m}\right), u(t) \in \rho \cdot U\right\}, \rho \geq 0$. It is easily seen that the corresponding trajectories coincide with the trajectories $\varphi^{\rho}(t, x, u)$ of

$$
\dot{x}(t)=f^{\rho}(x(t), u(t))=f(x(t), \rho u(t)), u \in \mathcal{U} .
$$

Clearly, this is a special case of a parameter-dependent control system as considered above. The maximal chain transitive sets $E_{i}^{0}$ of the uncontrolled system are contained in chain control sets $E_{i}^{\rho}$ of the $\rho$-system for every $\rho>$ 0 . Their lifts $\mathcal{E}_{i}^{\rho}$ are the maximal chain transitive sets of the corresponding control flows $\Phi^{\rho}$. Every chain transitive set for small positive $\rho>0$ is of this form with a unique $E_{i}^{0}, i=1, \ldots, m$ (see [8]). For larger $\rho$-values, there may exist further maximal chain transitive sets $\mathcal{E}^{\rho}$ containing no chain transitive set of the unperturbed system. An easy example is obtained by looking at systems where for some $\rho_{0}>0$ a saddle node bifurcation occurs in $\dot{x}=f(x, \rho)$. A more intricate example is [8], Example 4.7.8. Observe that for larger $\rho$-values the chain control sets may intersect and hence coincide. From Theorem 1 we obtain that the maps

$$
\rho \mapsto \operatorname{cl} D^{\rho} \text { and } \rho \mapsto E^{\rho}
$$


are left and right continuous, respectively. We call $(u, x) \in \mathcal{U} \times \mathbb{R}^{d}$ an inner pair, if there is $T>0$ with $\varphi(T, x, u) \in \operatorname{int} \mathcal{O}^{+}(x)$. The following $\rho$-inner pair condition will be relevant:

For all $\rho^{\prime}, \rho \in\left[\rho_{*}, \rho^{*}\right)$ with $\rho^{\prime}>\rho$ and all chain control sets $E^{\rho}$ every $(u, x) \in \mathcal{E}^{\rho}$ is an inner pair for $(2)^{\rho^{\prime}}$.

By [8, Corollary 4.1.12] the $\rho$-inner pair condition and local accessibility imply that for increasing families of control sets $D^{\rho}$ and chain control sets $E^{\rho}$ with $D^{\rho} \subset E^{\rho}$ the number of discontinuity points of (5) is at most countable; they coincide for both maps and at common continuity points $\mathrm{cl} D^{\rho}=E^{\rho}$. The number of discontinuity points may be dense (without the inner pair condition, there may be "large" discontinuities which persist for all $\rho>0$ ).

Remark 3. The inner-pair condition (6) may appear unduly strong. However it is easily verified for small $\rho>0$ if the unperturbed system has a controllable linearization (more information is given in [8], Chapter 4.) For general $\rho>0$ the inner pair condition holds, e.g., for coupled oscillators if the number of controls is equal to the degrees of freedom; for this result and more general conditions see Gayer [14].)

Next we show that the number of discontinuity points with a lower bound on the discontinuity size is finite in every compact $\rho$-interval. Thus, from a practical point of view, only finitely many may be relevant.

Lemma 1. Consider families of increasing control sets $D^{\rho}$ and chain control sets $E^{\rho}$ with $D^{\rho} \subset E^{\rho}$.

(i) Let $\rho_{0} \geq 0$ and assume that $E^{\rho_{0}} \subset \operatorname{cl} D^{\rho}$ for $\rho>\rho_{0}$. For every $\varepsilon>0$ there is $\delta>0$ such that for all $\rho>\rho_{0}$

$$
\rho-\rho_{0}<\delta \text { implies } \mathrm{d}_{H}\left(\operatorname{cl} D^{\rho}, E^{\rho}\right)<\varepsilon .
$$

(ii) Let $\rho_{0}>0$ and assume that $E^{\rho} \subset \operatorname{cl} D^{\rho_{0}}$ for $\rho<\rho_{0}$. For every $\varepsilon>0$ there is $\delta>0$ such that for all $\rho<\rho_{0}$

$$
\rho_{0}-\rho<\delta \text { implies } \mathrm{d}_{H}\left(\operatorname{cl} D^{\rho}, E^{\rho}\right)<\varepsilon .
$$

Proof. (i) Since for all $\rho$ the inclusion $\operatorname{cl} D^{\rho} \subset E^{\rho}$ holds, one only has to show that

$$
\operatorname{dist}\left(E^{\rho}, \operatorname{cl} D^{\rho}\right)=\sup \left\{d\left(x, \operatorname{cl} D^{\rho}\right), x \in E^{\rho}\right)<\varepsilon .
$$

Let $\varepsilon>0$. By right continuity of $\rho \mapsto E^{\rho}$ there is $\delta>0$ such that $\operatorname{dist}\left(E^{\rho}, E^{\rho_{0}}\right)<\varepsilon$ for all $\rho$ with $\delta>\rho-\rho_{0}>0$ and we know that $E^{\rho_{0}} \subset \operatorname{cl} D^{\rho} \subset E^{\rho}$. Thus

$$
\begin{aligned}
\operatorname{dist}\left(E^{\rho}, \operatorname{cl} D^{\rho}\right) & =\sup \left\{d\left(x, \operatorname{cl} D^{\rho}\right), x \in E^{\rho}\right\} \\
& \leq \sup \left\{d\left(x, E^{\rho_{0}}\right), x \in E^{\rho}\right\} \\
& =\operatorname{dist}\left(E^{\rho}, E^{\rho_{0}}\right)<\varepsilon .
\end{aligned}
$$


(ii) Similarly, left continuity of $\rho \mapsto \operatorname{cl} D^{\rho}$ yields for $\varepsilon>0$ a number $\delta>0$ such that $d_{H}\left(\operatorname{cl} D^{\rho_{0}}, \operatorname{cl} D^{\rho}\right)=\operatorname{dist}\left(\operatorname{cl} D^{\rho_{0}}, \operatorname{cl} D^{\rho}\right)<\varepsilon$ for all $\rho$ with $-\delta<\rho-\rho_{0}<0$ and we know that $E^{\rho} \subset \operatorname{cl} D^{\rho_{0}}$. Thus

$$
\begin{aligned}
\operatorname{dist}\left(E^{\rho}, \operatorname{cl} D^{\rho}\right) & =\sup \left\{d\left(x, \operatorname{cl} D^{\rho}\right), x \in E^{\rho}\right\} \\
& \leq \sup \left\{d\left(x, \operatorname{cl} D^{\rho}\right), x \in \operatorname{cl} D^{\rho_{0}}\right\} \\
& =\operatorname{dist}\left(\operatorname{cl} D^{\rho_{0}}, \operatorname{cl} D^{\rho}\right)<\varepsilon .
\end{aligned}
$$

Proposition 1. Suppose that the $\rho$-inner pair condition (6) holds on an interval $\left[\rho_{*}, \rho^{*}\right] \subset[0, \infty)$. Then for every $\varepsilon>0$ there are only finitely many points $\rho \in\left[\rho_{*}, \rho^{*}\right]$ where $d_{H}\left(\operatorname{cl} D^{\rho}, E^{\rho}\right) \geq \varepsilon$.

Proof. The inner pair condition guarantees that for all $\rho<\rho^{\prime}$ in $\left[\rho_{*}, \rho^{*}\right]$ one has $E^{\rho} \subset \operatorname{cl} D^{\rho^{\prime}}$; see [8, Section 4.8]. Let $\varepsilon>0$. By the preceding lemma one finds for every point $\rho_{0} \in\left[\rho_{*}, \rho^{*}\right]$ a number $\delta>0$ such that for all $\rho \neq \rho_{0}$ in $U\left(\rho_{0}\right):=\left[\rho_{*}, \rho^{*}\right] \cap\left(\rho_{0}-\delta, \rho_{0}+\delta\right)$

$$
d_{H}\left(\operatorname{cl} D^{\rho}, E^{\rho}\right)<\varepsilon .
$$

Now compactness implies that $\left[\rho_{*}, \rho^{*}\right]$ is covered by finitely many of these sets $U\left(\rho_{0}\right)$. Only their midpoints $\rho_{0}$ may have $d\left(\operatorname{cl} D^{\rho_{0}}, E^{\rho_{0}}\right) \geq \varepsilon$.

Remark 4. The same arguments show that the reachable sets enjoy the same properties, if their closures are compact and the $\rho$-inner pair condition holds everywhere.

\section{Bifurcation from a singular point}

In this section we discuss bifurcation of control sets and chain control sets from a singular point $x^{0}$. Here the linearized system is a (homogeneous) bilinear control system; the associated control flow is a linear skew product flow over the base of control functions.

Assume that $x^{0} \in \mathbb{R}^{d}$ remains fixed for all $\alpha$ and for all controls, i.e.,

$$
f_{i}\left(\alpha, x^{0}\right)=0 \text { for } i=0,1, \ldots, m \text {. }
$$

Then the system linearized with respect to $x$ is

$$
\dot{y}(t)=\left[A_{0}+\sum_{i=1}^{m} u_{i}(t) A_{i}\right] y(t), u \in \mathcal{U},
$$

where $A_{i}:=D f_{i}\left(x^{0}\right)$. The solutions are $y\left(t, y^{0}, u\right)=D_{2} \varphi\left(t, x^{0}, u\right)\left(y^{0}\right)$ and the associated linearized control flow has the form

$$
\mathbf{T} \Phi_{t}\left(x^{0}\right): \mathcal{U} \times \mathbb{R}^{d} \rightarrow \mathcal{U} \times \mathbb{R}^{d},(u, y) \rightarrow\left(\theta_{t} u, y\left(t, y^{0}, u\right)\right) .
$$


Clearly this flow is linear in the fibers $\{u\} \times \mathbb{R}^{d}$, since it corresponds to linear homogeneous differential equations.

The singular point is a trivial control set which is invariant. It need not be a chain control set. The bifurcation from this control set can be analyzed using the Lyapunov exponents of the linearized system which are given by

$$
\lambda\left(u, y^{0}\right)=\lim \sup _{t \rightarrow \infty} \frac{1}{t} \log \left\|y\left(t, y^{0}, u\right)\right\| .
$$

We note that there are a number of closely related notions for this generalization of the real parts of eigenvalues to nonautonomous linear differential equations. Basic results are given by Johnson, Palmer, and Sell [20]; see [8, Section 5.5] for some additional information.

The following result due to Grünvogel [17] shows that control sets near the singular point are determined by the Lyapunov exponents; note that for periodic controls, the Lyapunov exponents are just the Floquet exponents.

Theorem 2. Consider the control-affine systems (2) with a singular point $x^{0} \in \mathbb{R}^{d}$ satisfying (7) and assume that the accessibility rank condition (4) holds for all $x \neq x^{0}$. Furthermore assume that

(i) there are periodic control functions $u^{s}$ and $u^{h}$ such that for $u^{s}$ the linearized system is exponentially stable, i.e., the corresponding Lyapunov exponents satisfy

$$
0>\lambda_{1}^{s}>\ldots>\lambda_{d}^{s}
$$

and for $u^{h}$ the corresponding Lyapunov exponents satisfy

$$
\lambda_{1}^{h} \geq \ldots \geq \lambda_{k}^{h}>0>\lambda_{k+1}^{h}>\ldots>\lambda_{d}^{h} ;
$$

(ii) all pairs $\left(u^{h}, x\right) \in \mathcal{U} \times \mathbb{R}^{d}$ with $x \neq x^{0}$ are strong inner pairs, i.e., $\varphi\left(t, x, u^{h}\right) \in \operatorname{int} \mathcal{O}^{+}(x)$ for all $t>0$.

Then there exists a control set $D$ with nonvoid interior such that $x^{0} \in \partial D$.

Using this result one observes in a number of control systems, e.g., in the Duffing-Van der Pol oscillator [17], that for some $\alpha$-values the singular point $x^{0}$ is exponentially stable for all controls, hence there are no control sets near $x^{0}$. Then, for increasing $\alpha$-values, control sets $D^{\alpha}$ occur with $x^{0} \in \partial D^{\alpha}$. For some upper $\alpha$-value, they move away from $x^{0}$.

Remark 5. Assumption (i) in Theorem 2 is in particular satisfied, if 0 is in the interior of the highest Floquet spectral interval (cp. [8]) and the corresponding subbundle is one-dimensional.

Remark 6. Grünvogel [17] also shows that there are no control sets in a neighborhood of the origin if zero is not in the interior of the Morse spectrum of the linearized system (8). This also follows from a Hartman-Grobman Theorem for skew product flows; see Bronstein/Kopanskii [4]. One has to take into account that the spectral condition implies hyperbolicity, since the base space $\mathcal{U}$ is chain recurrent. Then the use of appropriate cut-off functions yields the desired local version. 
Remark \%. Using averaging techniques, Grammel/Shi [15] considered the stability behavior and the Lyapunov spectrum of bilinear control systems perturbed by a fast subsystem.

Remark 8. A number of questions remains open in this context: Is the control set containing the singular point $x^{0}$ on its boundary invariant? (certainly it is not closed.) Can one also prove a bifurcation of control sets if other spectral intervals (instead of the highest interval) contain 0? What happens if the corresponding subbundles are higher dimensional? Is the consideration of periodic controls necessary?

We see that a characteristic of bifurcations from a singular point is that there is (at least) one transitional state. Here the control set $\left\{x^{0}\right\}$ has already split into two or more control sets which, however, still form a single chain control set. The bifurcation is complete when also the chain control set has split. This should be compared with L. Arnold's bifurcation pattern for stochastic systems [1]; see in particular the discussion in Johnson, Kloeden, and Pavani [18].

The Hartman-Grobman Theorem alluded to in Remark 6 gives a topological conjugacy result. As for differential equations, a natural next step is to classify the bifurcation behavior by introducing normal forms of nonlinear systems based on smooth conjugacies. Since we discuss open loop systems with restricted control values, feedback transformations will not be allowed (thus this is different from classical concepts of normal forms in control theory). The admissible transformations have to depend continuously on the control functions $u$ in the base space $\mathcal{U}$ of the skew product flow. This makes it possible (see [11]) to use methods from normal forms of nonautonomous differential equations (Siegmund [25]). Then conjugacies eliminate all nonresonant terms in the Taylor expansion without changing the other terms up to the same order. We note that there is also related work in the theory of random dynamical systems which can be considered as skew product flows with an invariant measure on the base space; compare L. Arnold [1].

We consider the control affine system (2) and assume that $f_{0}, \ldots, f_{m}$ are $C^{k}$ vector fields for some $k \geq 2$. Then the associated control flow $\Phi$ is, for fixed $u \in \mathcal{U}, k$ times continuously differentiable with respect to $x$. Our notion of conjugacies which, naturally, depend on $u$ is specified in the following definition.

Definition 1. Let $\varphi: \mathbb{R} \times \mathcal{U} \times \mathbb{R}^{d} \rightarrow \mathbb{R}^{d}$ and $\psi: \mathbb{R} \times \mathcal{U} \times \mathbb{R}^{d} \rightarrow \mathbb{R}^{d}$ be two control systems of the form (2) with common singular point $x^{0}$. Then $\varphi$ and $\psi$ are said to be $C^{k}$ conjugate if there exists a bundle mapping

$$
\mathcal{U} \times \mathbb{R}^{d} \ni(u, x) \mapsto(u, H(x, u)) \in \mathcal{U} \times \mathbb{R}^{d}
$$

which preserves the zero section $\mathcal{U} \times\{0\}$, such that

(i) $x \mapsto H(x, u)$ is a local $C^{k}$ diffeomorphism (near $x^{0}=0$ ) for each fixed 
$u \in \mathcal{U}$ (with inverse denoted by $y \mapsto H(y, u)^{-1}$ ),

(ii) $(u, x) \mapsto H(x, u)$ and $(u, y) \mapsto H(y, u)^{-1}$ are continuous,

(iii) for all $t \in \mathbb{R}, x \in \mathbb{R}^{d}$ and $u \in \mathcal{U}$ the conjugacy

$$
\psi(t, u, H(x, u))=H\left(\theta_{t} u, \varphi(t, x, u)\right)
$$

holds.

Next we discuss the Taylor expansions and the terms which are to be eliminated by conjugacies. We rewrite system (2) in the form

$$
\dot{x}=A(u(t)) x(t)+F(x(t), u(t)),
$$

where the nonlinearity is given by

$$
F(x(t), u(t))=f_{0}(x(t))-A_{0} x(t)+\sum_{i=1}^{m} u_{i}(t)\left(f_{i}(x(t))-A_{i} x(t)\right) .
$$

In the following we assume that the linearized system is in block diagonal form and that the nonlinearity is $C^{k}$-bounded. More precisely we assume $A=\operatorname{diag}\left(A^{(1)}, \ldots, A^{(n)}\right)$ with $A^{(i)}: U \rightarrow \mathbb{R}^{d_{i} \times d_{i}}, d_{1}+\cdots+d_{n}=d$ and $\left\|D_{x}^{i} F\left(x^{0}, u\right)\right\| \leq M$ for $i=1, \ldots, k, u \in \mathcal{U}$, with a constant $M>0$.

The block diagonalization of the linearized system into the matrices $A^{(i)}$ corresponds to a decomposition of $\mathbb{R}^{d}$ into $d_{i}$-dimensional subspaces. Corresponding to the block diagonal structure of $A$ one can write $x=$ $\left(x^{(1)}, \ldots, x^{(n)}\right) \in \mathbb{R}^{d}$ and $F=\left(F^{(1)}, \ldots, F^{(n)}\right)$ with the component functions $F^{(i)}: \mathbb{R}^{d} \times U \rightarrow \mathbb{R}^{d_{i}}$. For a multi-index $\ell=\left(\ell_{1}, \ldots, \ell_{n}\right) \in \mathbb{N}_{0}^{\ell}$ let $|\ell|=\ell_{1}+\cdots+\ell_{n}$ denote the order and define

$$
D_{x}^{\ell} F=D_{x^{(1)}}^{\ell_{1}} \cdots D_{x^{(n)}}^{\ell_{n}} F \quad \text { and } \quad x^{\ell}=\underbrace{x^{(1)} \cdots x^{(1)}}_{\ell_{1} \text {-times }} \cdots \underbrace{x^{(n)} \cdots x^{(n)}}_{\ell_{n} \text {-times }} .
$$

Now we can expand $F(\cdot, u(t))$ into a Taylor series at $x^{0}$

$$
F(x, u(t))=\sum_{\ell \in \mathbb{N}_{0}^{n}: 2 \leq|\ell| \leq k} \frac{1}{\ell !} D_{x}^{\ell} F\left(x^{0}, u(t)\right) \cdot\left(x-x^{0}\right)^{\ell}+o\left(\left\|x-x^{0}\right\|^{k}\right),
$$

where $\ell !=\ell_{1} ! \cdots \ell_{n}$ !. For simplicity we assume without loss of generality that $x^{0}=0$. We are looking for a condition which ensures the existence of a $C^{k}$ conjugacy which eliminates the $j$-th component $D_{x}^{\ell} F^{(j)}(0, u(t)) \cdot x^{\ell}$ of a summand in the Taylor expansion of $F$.

Let $\Phi=\operatorname{diag}\left(\Phi^{(1)}, \ldots, \Phi^{(n)}\right)$ denote the solution of the linearized system (8), i.e., $\Phi^{(i)}(t, u) y^{(i)}$ solves the control system

$$
\dot{y}^{(i)}(t)=A^{(i)}(u(t)) y^{(i)}(t) \quad \text { in } \mathbb{R}^{d_{i}}, u \in \mathcal{U},
$$


with $\Phi^{(i)}(0, u) y^{(i)}=y^{(i)}$. The nonresonance condition will be based on exponential dichotomies: we associate to each $\Phi^{(i)}$ an interval $\Lambda_{i}=\left[a_{i}, b_{i}\right]$ such that for every $\varepsilon>0$ there is $K>0$ with

$$
\left\|\Phi^{(i)}(s, u)^{-1}\right\| \leq K e^{-\left(a_{i}-\varepsilon\right) s} \text { and }\left\|\Phi^{(i)}(s, u)\right\| \leq K e^{\left(b_{i}+\varepsilon\right) s}
$$

for $s \geq 0, u \in \mathcal{U}$.

Remark 9. Condition (11) holds if we define, for the Lyapunov exponents as in (9),

$$
a_{i}=\inf \left\{\lambda\left(u, y^{(i)}\right),\left(u, y^{(i)}\right) \in \mathcal{U} \times \mathbb{R}^{d_{i}}\right\}, b_{i}=\sup \left\{\lambda\left(u, y^{(i)}\right),\left(u, y^{(i)}\right) \in \mathcal{U} \times \mathbb{R}^{d_{i}}\right\} .
$$

Then $\left[a_{i}, b_{i}\right]$ contains the dynamical spectrum of the corresponding system on $\mathbb{R}^{d_{i}}$ and the assertion follows from its properties; see [24] or [8, Section 5.4].

Next we state the normal form theorem for control systems at a singular point. It shows that nonresonant terms in the Taylor expansion can be eliminated without changing the other coefficients up to the same order. The proof of this theorem is given in [11], where also some first examples are indicated.

For compact sets $K_{1}, K_{2} \subset \mathbb{R}$ and integers $\ell_{1}, \ell_{2} \in \mathbb{Z}$ we define the compact set $\ell_{1} K_{1}+\ell_{2} K_{2}:=\left\{\ell_{1} k_{1}+\ell_{2} k_{2}: k_{i} \in K_{i}\right\}$ and we write $K_{1}<K_{2}$ iff $\max K_{1}<\min K_{2}$ and similarly for $K_{1}>K_{2}$.

Theorem 3. Consider a class of $C^{k}$ control affine systems (2) satisfying the assumptions above. Suppose that to each block an interval $\Lambda_{i}$ is associated with property (11). Let $\ell=\left(\ell_{1}, \ldots, \ell_{n}\right) \in \mathbb{N}_{0}^{n}$ be a multi-index of order $2 \leq|\ell| \leq k$ and assume that for some $j$ the nonresonance condition

$$
\Lambda_{j}<\sum_{i=1}^{n} \ell_{i} \Lambda_{i} \quad \text { or } \quad \Lambda_{j}>\sum_{i=1}^{n} \ell_{i} \Lambda_{i}
$$

holds. Then there exists a $C^{k}$ conjugacy between (10) and a system

$$
\dot{x}=A(u(t)) x(t)+G(x(t), u(t))
$$

which eliminates the $j$-th Taylor component $\frac{1}{\ell !} D_{x}^{\ell} F^{(j)}(0, u(t)) \cdot x^{\ell}$ belonging to the multi index $\ell$ and leaves fixed all other Taylor coefficients up to order $|\ell|$,

Remark 10. This result shows that-under a non-resonance condition-some terms can be eliminated without changing the other terms up to the same order. We would like to stress that higher order terms may be changed; no analysis of terms of arbitrarily high order appears feasible in this context.

Remark 11. The theorem above leads to the problem to find a complete catalogue of systems of order $k$ without terms which can be eliminated. Such an analysis must be made for every control range (i.e. for every base flow). It is an interesting question, when different control ranges may lead to the same normal forms. 
Remark 12. The Lyapunov exponents generalize the real part of eigenvalues. The imaginary parts of eigenvalues determine the rotational behavior and hence are also of relevance in describing the bifurcation behavior. For stochastic equations (where an ergodic invariant measure on the base space is given), Arnold/San Martin [2] and Ruffino [23] have discussed a corresponding rotation number. Another concept is used by Johnson and others for Hamiltonian skew product flows; in particular, the latter is used for a generalization [13] of a theorem due to Yakubovich who analyzed linear quadratic optimal control problems for periodic systems.

\section{Bifurcation from invariant control sets}

The previous section dealt with bifurcation of control sets from a singular point. Other singular scenarios where bifurcation phenomena occur are totally unexplored. The present section concentrates on the regular situation where local accessibility holds. Bifurcations will be considered for an invariant object in the state space (not for the more general case of invariant objects for the lifted system in $\mathcal{U} \times M)$.

A first question concerns the role of hyperbolicity in this regular context. In the theory of chaotic dynamical systems a classical tool is Bowen's shadowing lemma. It allows one to find close to $(\varepsilon, T)$-chains trajectories of a hyperbolic differential equation or discrete dynamical system. In the context of control flows, a generalization has been given in Colonius/Du [5]. The required hyperbolicity condition refers to the linearized system given by

$$
\dot{x}=f(x, u), \dot{y}=D_{1} f(x, u) y, u \in \mathcal{U}^{\rho} .
$$

Theorem 4. Suppose that the uncontrolled system $\dot{x}=f(x, 0)$ is hyperbolic on a compact chain transitive component $\mathcal{M}$ and assume local accessibility for all $\rho>0$. Furthermore assume that the chain control set $E^{\rho}$ containing $\mathcal{M}$ has nonvoid interior. Then for $\rho>0$, small enough, $E^{\rho}=\mathrm{cl} D^{\rho}$ for a control set $D^{\rho}$ with nonvoid interior.

Remark 13. Since control flows are special skew product flows, it may appear natural to ask if a shadowing lemma for general skew product flows can be used in this context. However, closing the gap between chain controllability and controllability also requires to close gaps in the base space. Here, in general, hyperbolicity which only refers to the fibers cannot be used. Thus the shadowing lemma for general discrete-time skew product flows by Meyer and Sell [22] can not be used since it excludes jumps in the base space.

In another direction one can analyze the behavior near a hyperbolic equilibrium of the uncontrolled system. Then a natural question is if the local uniqueness of the equilibrium of the uncontrolled system transfers to the controlled system. A positive answer has been given in Remark 6 for the 
case of a singular equilibrium. The following result from Colonius/Spadini [12] gives an analogous result in the regular situation. For the formulation we need the notion of local control sets: For a subset $N \subset \mathbb{R}^{d}$ denote $\mathcal{O}_{N}^{+}(x)=\{\varphi(T, x, u), T>0, u \in \mathcal{U}$ and $\varphi(t, x, u) \in N$ for all $0 \leq t \leq T\}$. A subset $D \subset \mathbb{R}^{d}$ is called a local control set, if there exists a neighborhood $N$ of $\operatorname{cl} D$ such that $D \subset \operatorname{clO}_{N}^{+}(x)$ for all $x \in D$ and $D$ is maximal with this property.

Consider a parameter-dependent family of control systems

$$
\dot{x}(t)=f(\alpha, x(t), u(t)), u(t) \in \rho U,
$$

where $\alpha \in \mathbb{R}, \rho>0$ and $U \subset \mathbb{R}^{m}$ is bounded, convex and contains the origin in its interior. We consider the behavior near an equilibrium of the uncontrolled system with $\alpha=\alpha_{0}$ for small control range.

Theorem 5. Let $f: \mathbb{R} \times \mathbb{R}^{d} \times \mathbb{R}^{m} \rightarrow \mathbb{R}^{d}$ be a continuous map which is $C^{1}$ with respect to the last two variables. Consider a continuous family of equilibria $x_{\alpha} \in \mathbb{R}^{d}$ such that $f\left(\alpha, x_{\alpha}, 0\right)=0$ and assume that the pair of matrices $\left(D_{2} f\left(\alpha_{0}, x_{\alpha_{0}}, 0\right), D_{3} f\left(\alpha_{0}, x_{\alpha_{0}}, 0\right)\right)$ is controllable and $D_{2} f\left(\alpha_{0}, x_{\alpha_{0}}, 0\right)$ is hyperbolic. Then there exist $\varepsilon_{0}>0, \rho_{0}>0$ and $\delta_{0}>0$ such that, for all $\left|\alpha-\alpha_{0}\right|<\varepsilon_{0}$ and all $0<\rho<\rho_{0}$, the ball $\mathbf{B}\left(x_{\alpha_{0}}, \delta_{0}\right)$ contains exactly one local control set for (14) with parameter value $\alpha$.

Without hyperbolicity this claim is false. We proceed to a partial generalization of Grünvogel's theorem, Theorem 2. Since we discuss bifurcation from a nontrivial invariant set, here the direction of the unstable manifold will be important (it must be directed outward).

For an invariant control set $C$ with nonvoid interior and compact closure we denote the lift of $C$ by $\mathcal{C}$,

$$
\mathcal{C}=\operatorname{cl}\left\{(u, x) \in \mathcal{U} \times \mathbb{R}^{d}, \varphi(t, x, u) \in \operatorname{int} C \text { for all } t \in \mathbb{R}\right\} .
$$

The linearized flow over $\mathcal{C}$ is obtained by restricting attention to solutions of (13) with $(u, x, y) \in \mathcal{C} \times \mathbb{R}^{d}$. The corresponding Lyapunov exponents are

$$
\lambda(u, x, y)=\lim \sup _{t \rightarrow \infty} \frac{1}{t} \log \left|D_{2} \varphi(t, x, u) y(t)\right|
$$

For $x \in \partial C$ define the outer cone in $x$ for $C$ by

$$
\mathbf{K}_{x} C=\left\{y \in \mathbb{R}^{d}, \begin{array}{c}
\text { there are } \beta, \lambda_{0}>0 \text { such that for all } z \in \mathbb{R}^{d} \text { with } \\
|z-y|<\beta \text { and all } 0<\lambda<\lambda_{0} \text { one has } x+\lambda z \notin C
\end{array}\right\} .
$$

Theorem 6. Assume that the system is locally accessible and let $C$ be an invariant control set with nonvoid interior and compact closure. Assume that there exists a compact invariant subset $\mathcal{J} \subset \mathcal{C}$ with the following properties: (i) The unstable part of $\mathcal{J}$ is nontrivial, i.e., there is a subbundle decomposition of the vector bundle $\mathcal{J} \times \mathbb{R}^{d}$ into subbundles $\mathcal{V}^{-} \neq 0$ and $\mathcal{V}^{+}$, which are invariant under the linearized flow and exponentially separated, such that 


$$
\mathcal{J} \times \mathbb{R}^{d}=\mathcal{V}^{+} \oplus \mathcal{V}^{-}
$$

all Lyapunov exponents attained in $\mathcal{V}^{-}$are positive, and there are constants $\gamma, c>0$ with

$$
\mathbf{T} \Phi_{t}\left(u, x, y^{-}\right)<c \exp (\gamma t) \mathbf{T} \Phi_{t}\left(u, x, y^{+}\right) \text {for }\left(u, x, y^{ \pm}\right) \in \mathcal{V}^{ \pm},
$$

(ii) There is $\left(u, x, y^{-}\right) \in \mathcal{V}^{-}$such that $(u, x) \in \mathcal{J}$ and $x \in \partial C$ and $y^{-} \in \mathbf{K}_{x} C$, the outer cone in $x$ for $C$.

Then the invariant control set $C$ is a proper subset of the chain control set $E$ containing it.

Proof. We will construct a chain controllable set which has nontrivial intersections with $C$ and the complement of $C$. This implies the assertion. Consider the point $x$ as specified in the second assumption. Since $x$ is in the boundary of $C$, there are $v \in \mathcal{U}, \tau>0$, and a neighborhood $N$ of $x$ such that for all $z \in N$ one has $\varphi(\tau, z, v) \in \operatorname{int} C$. By an appropriately general version of the Unstable Manifold Theorem, see, e.g., [8, Section 6.4], our assumptions imply, that the set $\mathcal{J}$ has a nontrivial unstable manifold $\mathcal{W}^{-}$, which is Lipschitz close to $\mathcal{V}^{-}$. In particular, for $\left(u, x, y^{-}\right) \in \mathcal{V}^{-}$as specified in the assumptions, there is $x^{-} \in N \cap\left(\mathbb{R}^{d} \backslash C\right)$ with $x^{-} \in \mathcal{W}^{-}(u, x)$. Thus $d\left(\varphi\left(t, x^{-}, u\right), \varphi(t, x, u)\right) \rightarrow 0$ for $t \rightarrow-\infty$. Since $(u, x) \in \mathcal{J} \cap \mathcal{C}$ it follows that $\varphi(t, x, u) \in C$ for all $t \in \mathbb{R}$. By compactness of $C$, there are $z \in C$ and $t_{k} \rightarrow-\infty$ with

$$
\varphi\left(t_{k}, x, u\right) \rightarrow z \text { for } k \rightarrow \infty,
$$

Now fix $\varepsilon>0$ and $T>0$. We will construct a controlled $(\varepsilon, T)$-chain connecting $x^{-}$and $C$. Start in $x_{0}:=x^{-}$and define $u_{0} \in \mathcal{U}$ as the concatenation of $v$ with any control which keeps the trajectory in $C$ up to time $T$ and has the property that for some $T_{0}>T$ one has

$$
d\left(\varphi\left(T_{0}, x^{-}, u_{0}\right), z\right)<\frac{\varepsilon}{2} .
$$

There is $\tau>T$ such that

$$
d\left(\varphi\left(-\tau, x^{-}, u\right), z\right)<\frac{\varepsilon}{2} .
$$

Thus we define $x_{1}:=\varphi\left(-\tau, x^{-}, u\right), u_{1}:=u(-\tau+\cdot)$, and $T_{1}:=\tau$. This yields the desired $(\varepsilon, T)$ - chain from $x^{-}$to $x^{-}$hitting $C$.

The result above is only a first step, since it does not answer the question, if the gap between the control set $D$ and the chain control set $E \supset D$ is due to the presence of another control set sitting in $E$. A partial answer is given in the following result from [10] which shows when the loss of invariance is due to the merger with a variant control set. We need some preparations. Let $K \subset \mathbb{R}^{d}$ be compact and invariant. An attractor for the control flow $\Phi$ is a compact invariant set $A \subset \mathcal{U} \times K$ that admits a neighborhood $N$ such that 
$A=\omega(N)=\left\{(u, x) \in \mathcal{U} \times K\right.$, there are $t_{k} \rightarrow \infty$ and $\left(u_{k}, x_{k}\right) \in N$ with $\left.\Phi\left(t_{k}, u_{k}, x_{k}\right) \rightarrow(u, x)\right\}$. Define for chain recurrent components $\mathcal{E}, \mathcal{E}^{\prime}$

$$
\left[\mathcal{E}, \mathcal{E}^{\prime}\right]=\left\{(u, x) \in \mathcal{U} \times K, \omega^{*}(u, x) \subset \mathcal{E} \text { and } \omega(u, x) \subset \mathcal{E}^{\prime}\right\}
$$

here $\omega^{*}(u, x)$ denotes the limit set for $t \rightarrow-\infty$. The structure of attractors and their relation to chain control sets is described in the following proposition.

Proposition 2. Assume that for every $\rho>0$ every chain recurrent component $\mathcal{E}^{\rho}$ contains a chain recurrent component $E_{i}^{0}$ of the unperturbed system. Then there is $\rho_{0}>0$ such that for all $\rho$ with $\rho_{0}>\rho>0$ the attractors $\mathcal{A}^{\rho}$ of the $\rho$-system are given by

$$
\mathcal{A}^{\rho}=\bigcup_{i, j \in J}\left[\mathcal{E}_{j}^{\rho}, \mathcal{E}_{k}^{\rho}\right]
$$

where the allowed index sets $J$ coincide with those for $\rho=0$. The chain recurrent components $\mathcal{E}_{i}^{\rho}$ depend upper semicontinuously on $\rho$ and converge for $\rho \rightarrow 0$ toward $\mathcal{U} \times E_{i}^{0} ;$ all $\mathcal{E}_{j}^{\rho}$ are different and they have attractor neighborhoods of the form $\mathcal{U} \times B$ with $B \subset K$.

For a set $I \subset K$ the invariant domain of attraction is

$$
\mathbf{A}^{i n v}(I)=\left\{x \in K, \begin{array}{c}
\text { if } C \subset \operatorname{clO}^{+}(x) \text { is an invariant } \\
\text { control set, then } C \subset I
\end{array}\right\} .
$$

The invariant domain of attraction is closed and invariant.

For simplicity we assume that all control sets are in the interior of $K$. By local accessibility, all invariant control sets have nonvoid interiors.

We will assume that for all $\rho$ with $\rho_{1}>\rho>0$ the chain control sets $E_{i}^{\rho}$ are the closures of control sets $D_{i}^{\rho}$ with nonvoid interior; observe that some of the control sets in the attractor must be invariant, since every point can be steered into an invariant control set. Then $E_{i}^{\rho}=\mathrm{cl} D_{i}^{\rho}$ implies that also the lifts coincide, i.e., $\mathcal{E}_{i}^{\rho}=\mathcal{D}_{i}^{\rho}$. It follows that the attractors are given by

$$
\mathcal{A}^{\rho}=\bigcup_{i, j \in J}\left[\mathcal{D}_{i}^{\rho}, \mathcal{D}_{j}^{\rho}\right]
$$

We will analyze the case where for $\rho=\rho_{1}$ the set $\mathcal{A}^{\rho_{1}}$ has lost the attractor property.

Theorem 7. Consider the control system (2) in $\mathbb{R}^{d}$ and assume that $K=$ $\mathrm{cl} \operatorname{int} K \subset \mathbb{R}^{d}$ is a compact and connected set which is invariant for the system with input range given by $\rho_{1} U$ with $\rho_{1}>0$. Assume that the following strong invariance conditions describing the behavior near the boundary of $K$ are satisfied:

(i) For all $x \in L$ there is $\varepsilon_{x}>0$ with $\mathrm{d}(\varphi(t, x, u), \partial K) \geq \varepsilon_{x}$ for all $u \in \mathcal{U}$ and $t \geq 0$.

(ii) There is $\varepsilon_{0}>0$ such that for all $x \in \operatorname{cl} L$ and $u \in \mathcal{U}$ 


$$
y=\lim _{k \rightarrow \infty} \varphi\left(t_{k}, x, u\right) \in L \text { for } t_{k} \rightarrow \infty \text { implies } \mathrm{d}(y, \partial K) \geq \varepsilon_{0} .
$$

Consider the invariant sets in $\mathcal{U}^{\rho} \times K$

$$
\mathcal{I}^{\rho}=\bigcup_{i, j \in J}\left[\mathcal{D}_{i}^{\rho}, \mathcal{D}_{j}^{\rho}\right]
$$

and assume that they are attractors for $\rho<\rho_{1}$ and that the projection $I^{\rho_{1}}$ to $\mathbb{R}^{d}$ of $\mathcal{I}^{\rho_{1}}$ intersects the boundary of its invariant domain of attraction defined in (15), i.e.,

$$
I^{\rho_{1}} \cap \partial \mathbf{A}^{i n v}\left(I^{\rho_{1}}\right) \neq \varnothing .
$$

Then every attractor containing $\mathcal{I}^{\rho_{1}}$ contains a lifted variant control set $\mathcal{D}^{\rho_{1}}$ with $D^{\rho_{1}} \cap I^{\rho_{1}}=\varnothing$.

This theorem shows that the loss of the attraction property due to increased input ranges is connected with the merger of the attractor with a variant control set $D^{\rho_{1}}$. Connections to Input-to-State Stability are discussed in $[10]$.

Remark 14. The abstract Hartman-Grobman Theorem from [4] can also be applied to the system over an invariant control set. Here, for the linearized system, the base space is the lift of the invariant control set. However, for parameter-dependent systems, this entails that the base space changes with the parameter. Hence it does not appear feasible to obtain results which yield conjugacy for small parameter changes. Here, presumably, normal hyperbolicity assumptions are required.

Remark 15. Consider a family of increasing control set $D^{\rho}$ corresponding to increasing control ranges. Assume that they are invariant for $\rho \leq \rho_{0}$ and variant for $\rho>\rho_{0}$ Then Gayer [14] has shown that the map $\rho \mapsto \operatorname{cl} D^{\rho}$ has a discontinuity at $\rho_{0}$. This is a consequence of his careful analysis of the different parts of the boundary of control sets. This also allows him to describe in detail the merging of an invariant control set with a variant control set.

Finally we remark that the intuitive idea of a slowly varying bifurcation parameter is made more precise, if the bifurcation parameter actually is subject to slow variations. This leads to concepts of dynamic bifurcations. The fate of control sets for frozen parameters under slow parameter variations is characterized in Colonius/Fabbri [6].

\section{References}

1. L. ARnold, Random Dynamical Systems, Springer-Verlag, 1998.

2. L. Arnold And L. S. Martin, A multiplicative ergodic theorem for rotation numbers, J. Dynamics Diff. Equations, 1 (1989), pp. 95-119. 
3. M. V. Bebutov, Dynamical systems in the space of continuous functions, Dokl. Akad. Nauk SSSR, 27 (1940), pp. 904-906. in Russian.

4. I. U. Bronstein And A. Y. Kopanski, Smooth Invariant Manifolds and Normal Forms, World Scientific, 1994.

5. F. Colonius AND W. Du, Hyperbolic control sets and chain control sets, J. Dynamical and Control Systems, 7 (2001), pp. 49-59.

6. F. COlOnius AND R. FABBRI, Controllability for systems with slowly varying parameters, ESAIM: Control, Optimisation and Calculus of Variations, 9 (2003), pp. 207-216.

7. F. Colonius And L. Grüne, eds., Dynamics, Bifurcations and Control, Springer-Verlag, 2002.

8. F. Colonius and W. Kliemann, The Dynamics of Control, Birkhäuser, 2000.

9. - Mergers of control sets, in Proceedings of the Fourtheenth International Symposium on the Mathematical Theory of Networks and Systems (MTNS), Perpignan, France, June 19-23 2000, A. El Jai and M. Fliess, eds., 2000.

10. —_ Limits of input-to-state stability, Systems Control Lett., (2003). to appear.

11. F. Colonius And S. Siegmund, Normal forms for control systems at singular points, J. Dynamics Diff. Equations, (2003). to appear.

12. F. Colonius AND M. Spadini, Uniqueness of local control sets, 2002. submitted to J. Dynamical and Control Systems.

13. R. FABbri, R. Johnson, And C. NÚnez, On the Yakubovich frequency theorem for linear non-autonomous processes, Discrete and Continuous Dynamical Systems, (2003). to appear.

14. T. GAYER, Controlled and perturbed systems under parameter variation, 2003. Dissertation, Universität Augsburg, Augsburg, Germany.

15. G. Grammel And P. Shi, On the asymptotics of the Lyapunov spectrum under singular perturbations, IEEE Trans. Aut. Control, 45 (2000), pp. 565-568.

16. L. GRÜNE, Asymptotic Behavior of Dynamical and Control Systems under Perturbation and Discretization, Springer-Verlag, 2002.

17. S. Grünvogel, Lyapunov exponents and control sets, J. Diff. Equations, 187 (2003), pp. 201-225.

18. R. Johnson, P. Kloeden, And R. Pavani, Two-step transition in nonautonomous bifurcations: An explanation, Stochastic and Dynamics, 2 (2002), pp. 67-92.

19. R. Johnson And M. Nerurkar, Controllability, Stabilization, and the Regulator Problem for Random Differential Systems, vol. 136, No. 646 of Memoirs of the AMS, Amer. Math. Soc., 1998.

20. R. A. Johnson, K. J. Palmer, And G. R. Sell, Ergodic properties of linear dynamical systems, SIAM J. Math. Anal., 18 (1987), pp. 1-33.

21. W. KANG, Bifurcation and normal form of nonlinear control systems - part I and part II, SIAM J. Control Optim., 36 (1998), pp. 193-212 and 213-232.

22. K. R. Meyer And G. R. Sell, Melnikov transforms, Bernoulli bundles and almost periodic perturbations, Trans. Amer. Math. Soc., 129 (1989), pp. 63-105.

23. P. Ruffino, Rotation numbers for stochastic dynamical systems, Stochastics and Stochastics Reports, 60 (1997), pp. 289-318.

24. R. J. SACKER AND G. R. SELL, A spectral theory for linear differential systems, J. Diff. Equations, 27 (1978), pp. 320-358.

25. S. Siegmund, Normal forms for nonautonomous differential equations, J. Diff. Equations, 14 (2002), pp. 243-258. 